\title{
Using Support Vector Machines as an Intelligent Algorithm for Detecting Seizures from E EG Signals
}

\author{
Mojtaba Mohammadpoor*, Atefe Alizadeh
}

Department of Electrical and Computer Engineering, Gonabad Higher Education Complex, Gonabad, Iran

\section{A BSTRACT}

Introduction: Electroencephalography (EEG) is the mos t commonly used method to study the function of the brain. This study represents a computerized model for dis tinguishing between epileptic and healthy subjects using EEG signals with relatively high accuracy. Materials and Methods: The EEG database used in this study was obtained from the data available in Andrzejak. This dataset consists of 5 EEG sets (designated as A to E), each containing 100 EEG sections. Collections A and B comprised EEG signals that have been taken from 5 healthy volunteers. The $\mathrm{C}$ and $\mathrm{D}$ sets referred to EEGs from patients with: focal epilepsy (without ictal recordings) and the E set was derived from a patient with ictal: recording. Support vector machines were used after applying principal components analysis or: linear discriminant analysis over the features of the signals. MATLAB has been used to implement: and test the proposed classification algorithm. To evaluate the proposed method, the confusion: matrix, overall success rate, ROC, and the AUC of each class were extracted. K-fold cross-validation technique was used to validate the results. Results: The overall success rate achieved: in this study was above $82 \%$. Dimension reduction algorithms can improve its accuracy and: speed. Conclusion: It is helpful to be able to predict the occurrence of a seizure early and accurately. Using the computerized model represented in this study could accomplish this goal.

*Corresponding Author: Mojtaba Mohammadpoor

Email: mohammadpur@gonabad.ac.ir 


\title{
استفاده از ماشينهاى بردار يشتيبانى بهعنوان يك الكوريتهم هوشمند براى تشخيص تشنج از

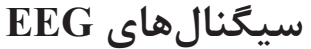

\author{
مجتبى محمديور"، عاطفه عليزاده
}

كروه مهيندسى برق و كامييوتر، مجتمع آموزش عالى كناباد، كناباد، ايران

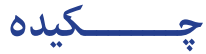

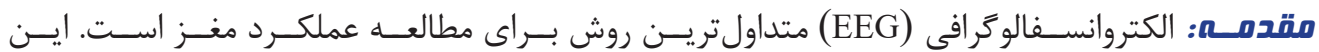

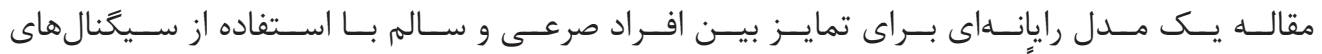

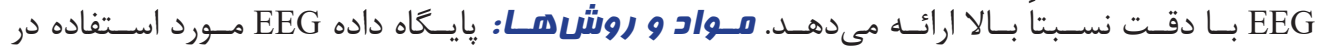

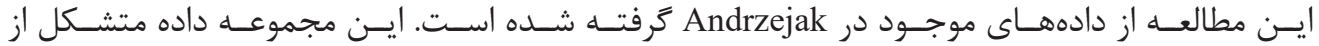

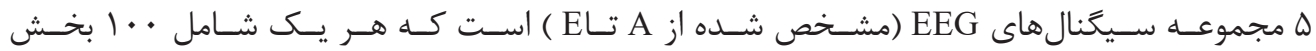

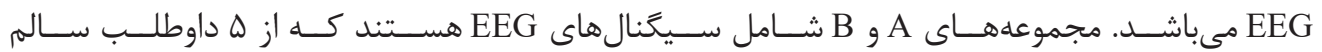

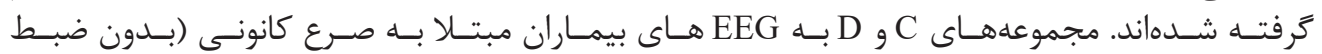

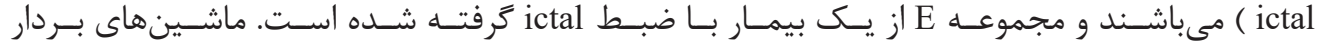

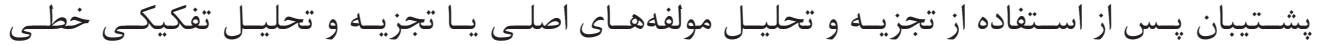

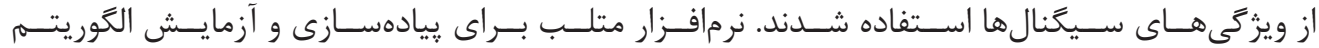

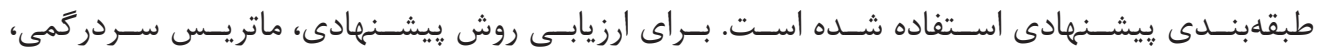

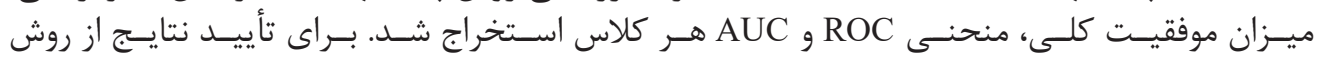

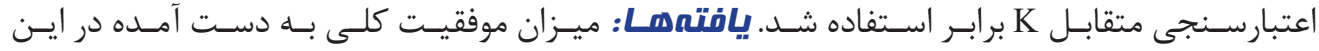

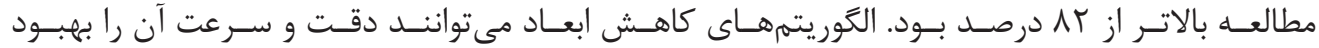

وازههاى كليدى:

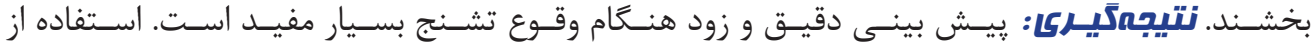

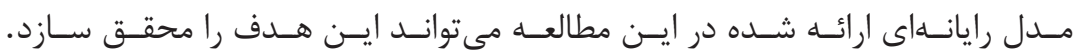

r- ب الكتروانسفالو r- بـ آنافيلاكسى يوستى منفعل
*ونيسنده مسئول: مجتبى محمديور

يست الكترونيك: mohammadpur@gonabad.ac.ir 


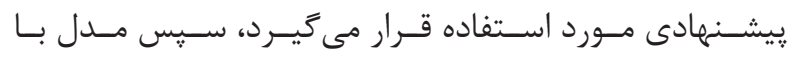

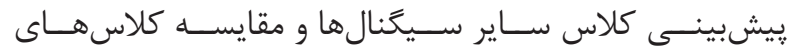

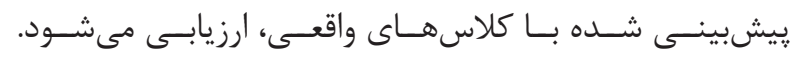

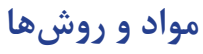

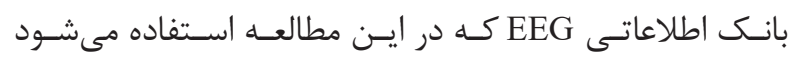

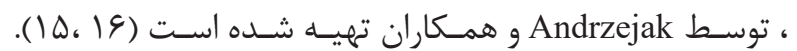

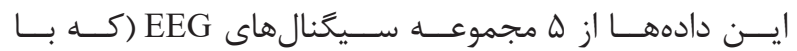

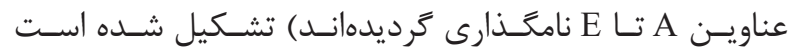

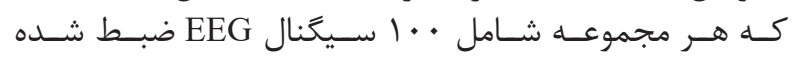

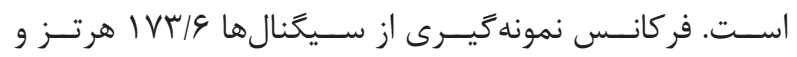

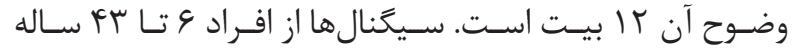

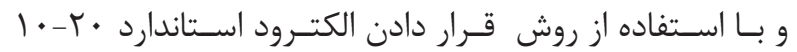

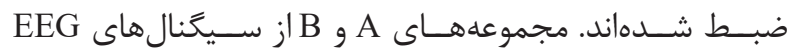

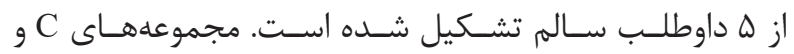

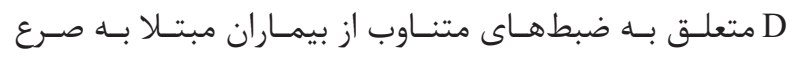

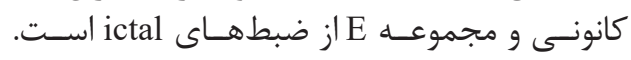

\section{استخراج ويزگى}

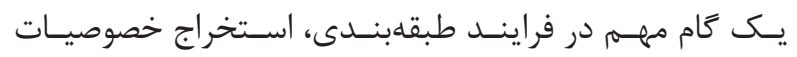

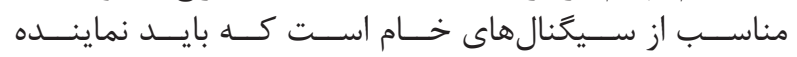

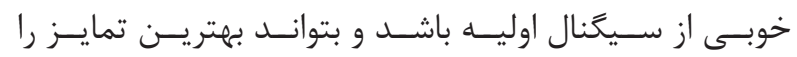

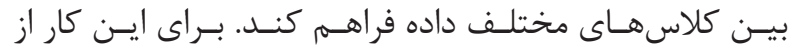
روشهـاى زيــر اسـتفاده كرديـهم.

\section{تبديل موجك كسسته}

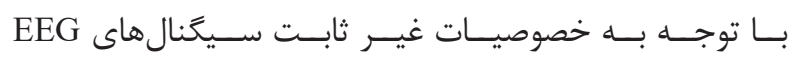

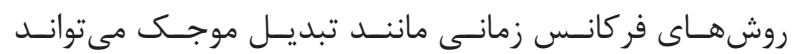

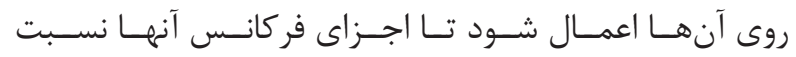

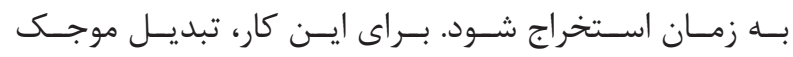

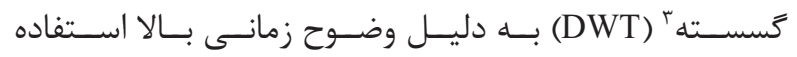

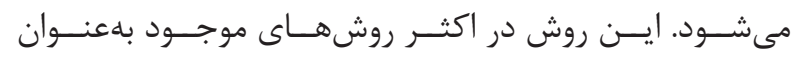

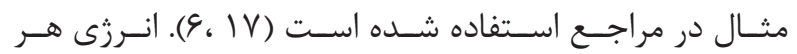

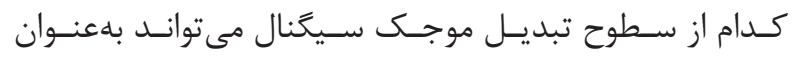

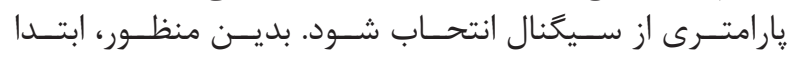

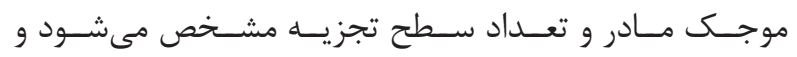

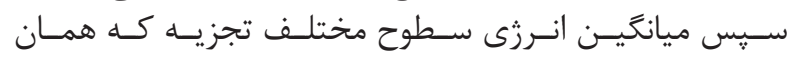

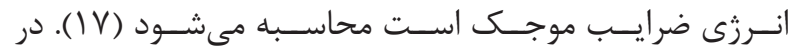

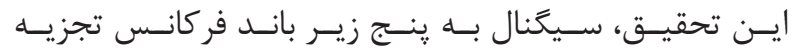

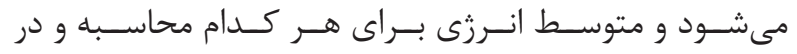

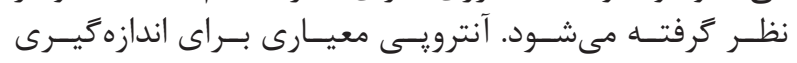

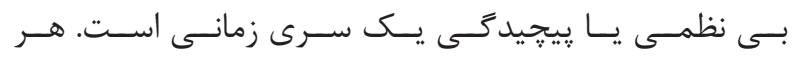

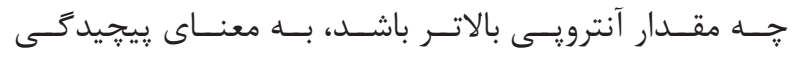

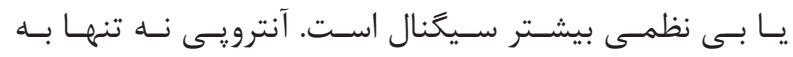

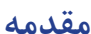

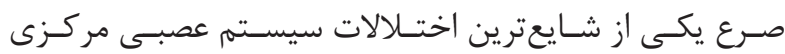

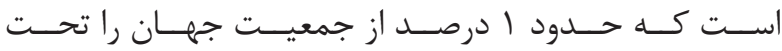

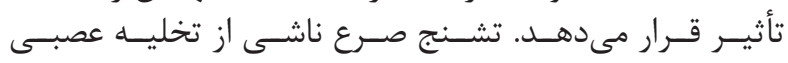

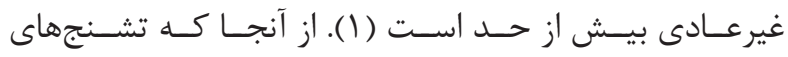

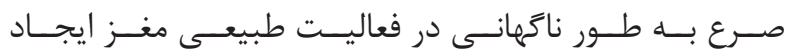

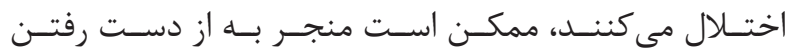

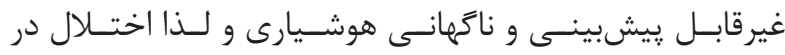

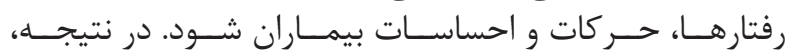

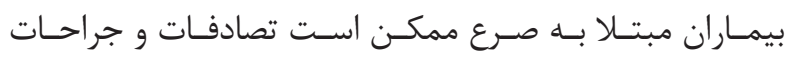

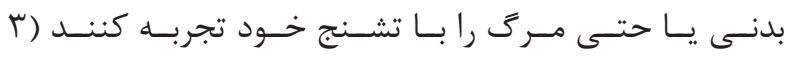

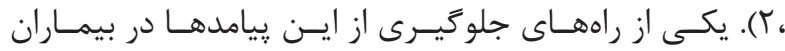

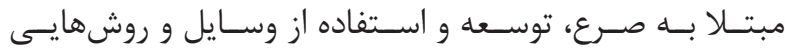

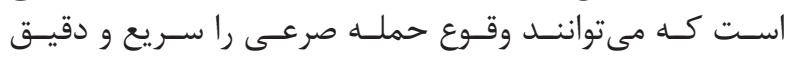

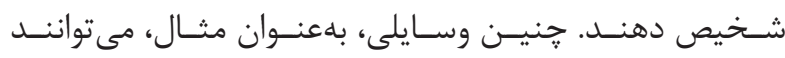

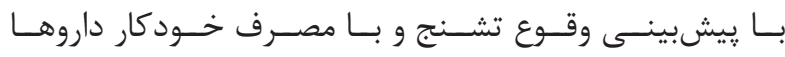

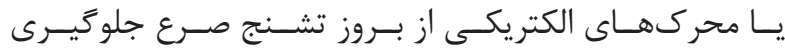

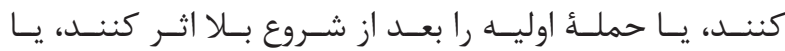

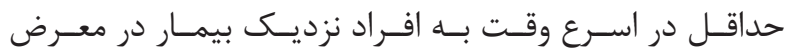

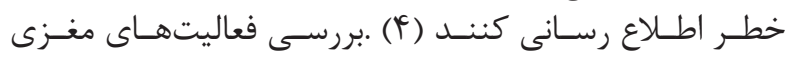

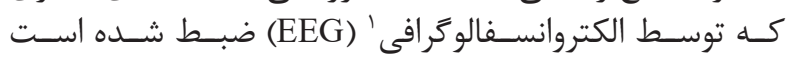

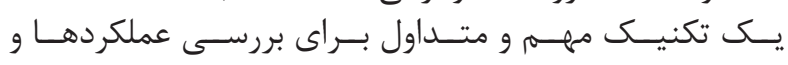

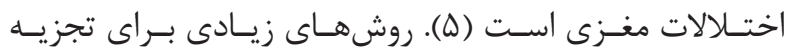

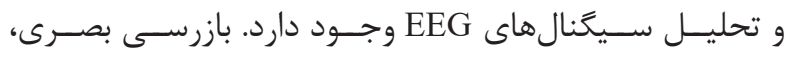

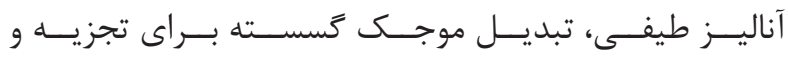

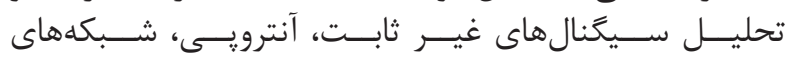

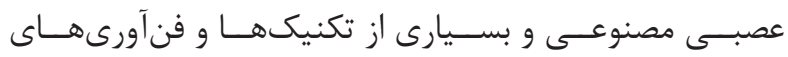

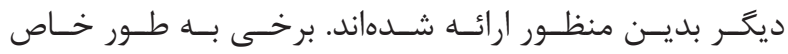

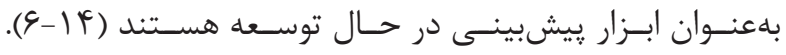

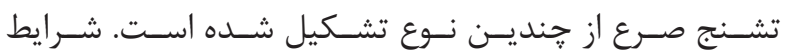

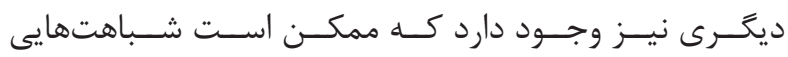

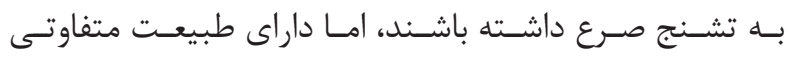

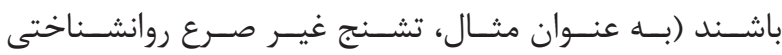

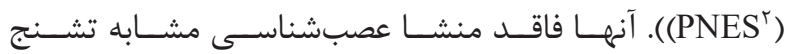

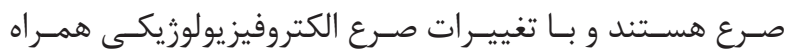

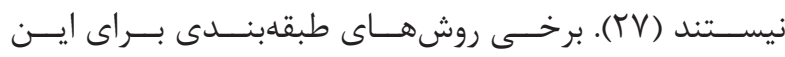

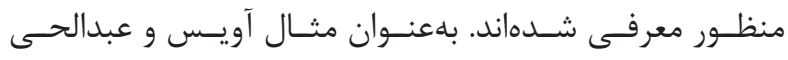

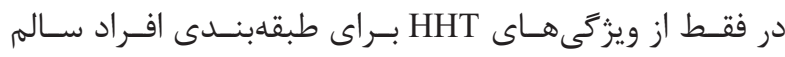

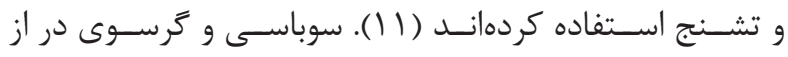

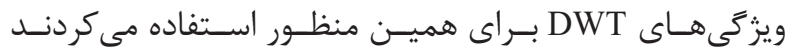

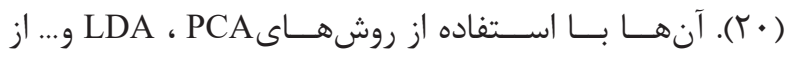

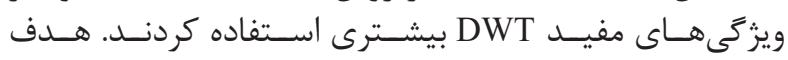

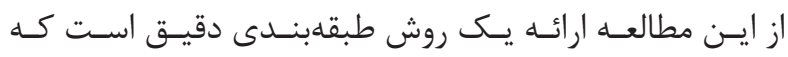

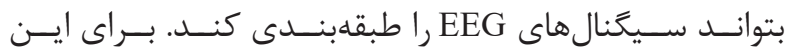

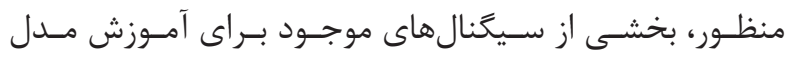

${ }^{3}$ Discrete Wavelet Transform 


\section{ماشين هاى بردار يشتيبان}

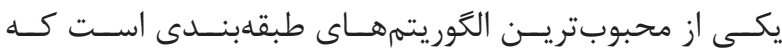

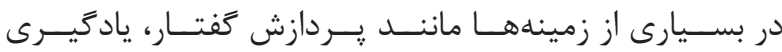

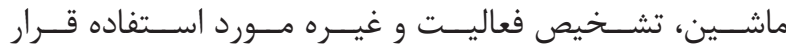

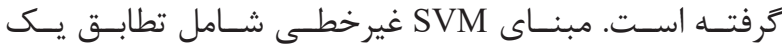

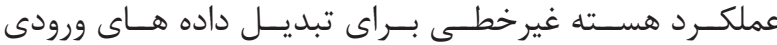

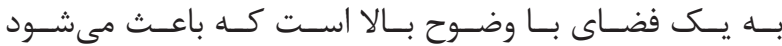

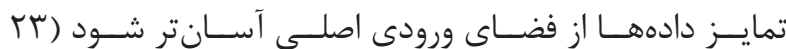
هT.

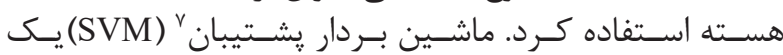

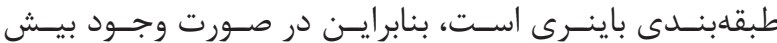

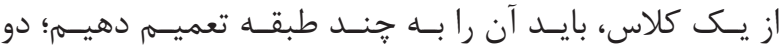

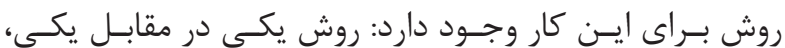

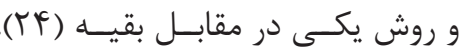

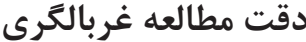

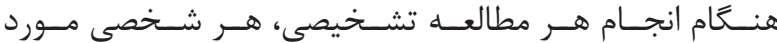

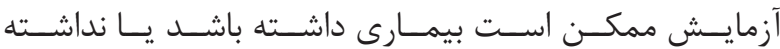

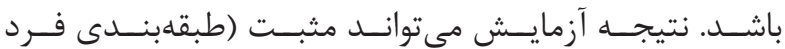

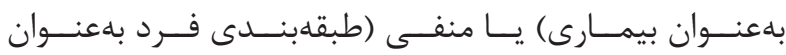

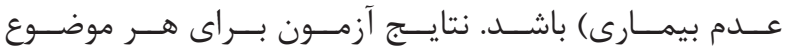

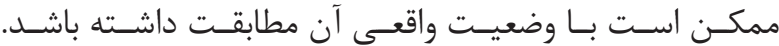

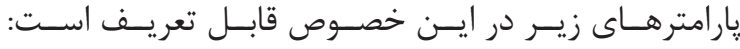
• مثبـتـت واقعىى (TP): افـراد بيمـار كـهـ بــه درسـتى بيمـار

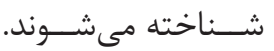

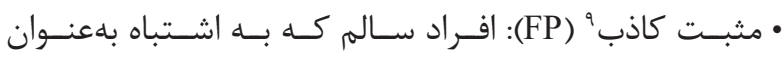

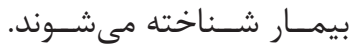

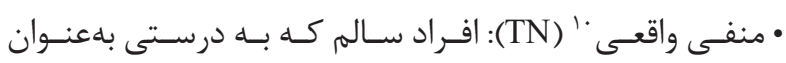

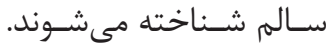

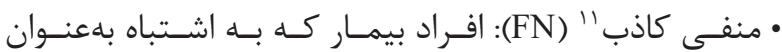

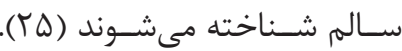

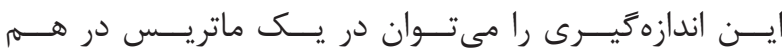

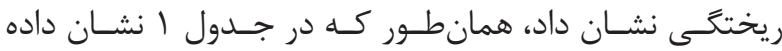

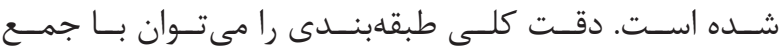

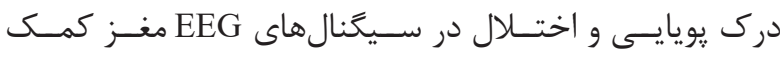

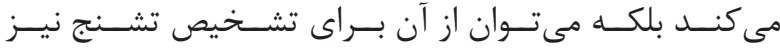

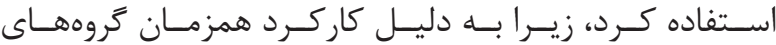

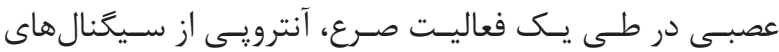
EEG

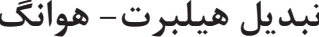

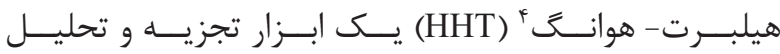

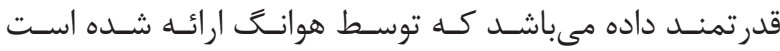

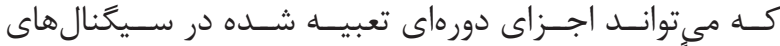

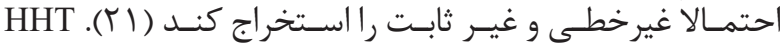

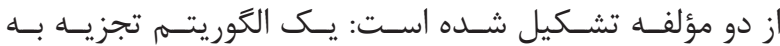

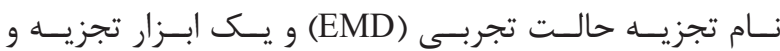

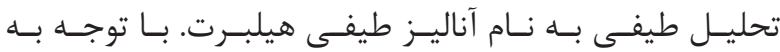

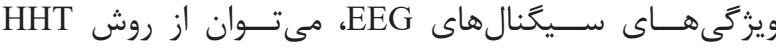

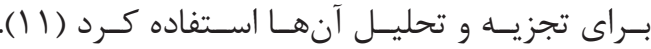

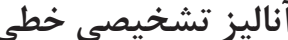

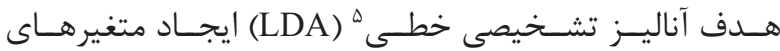

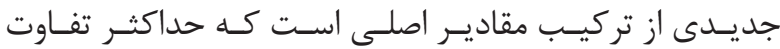

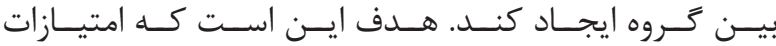

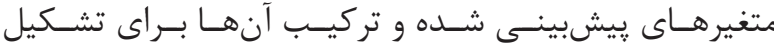

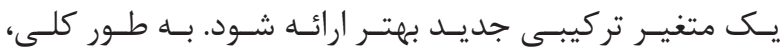

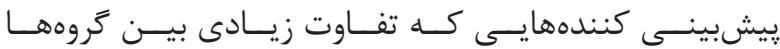

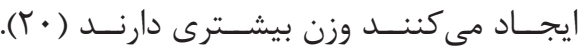

\section{تجزيه و تحليل مؤلفه اصلى}

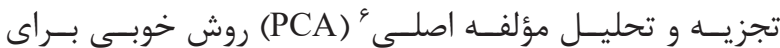

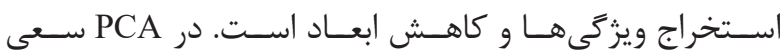

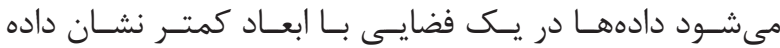

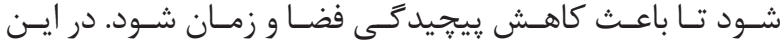

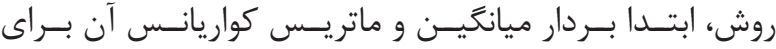

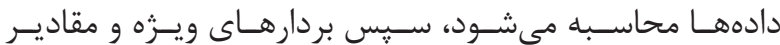

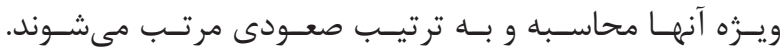

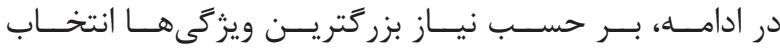

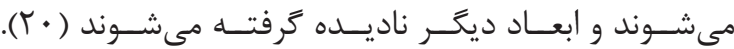

جدول ا- يارامترهاى ماتريس درهم ريختىى

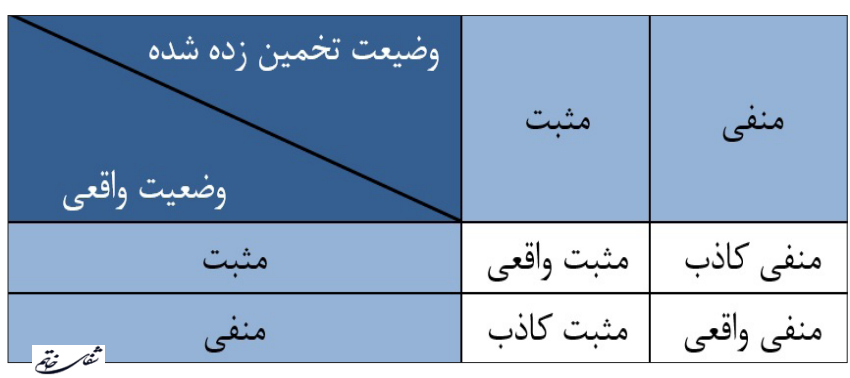

${ }^{4}$ Hilbert-Huang Transform

${ }^{5}$ Linear discriminate Analysis

${ }^{6}$ Principal component Analysis

${ }^{7}$ Support Vector Machines
${ }^{8}$ True Positive

${ }^{9}$ False positive

${ }^{10}$ True Negative

${ }^{11}$ False Negative 


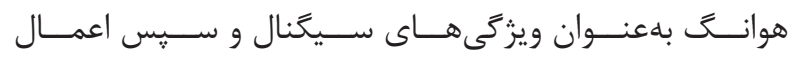

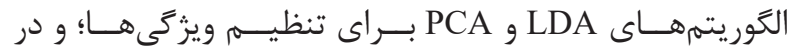

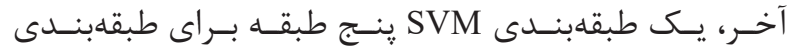

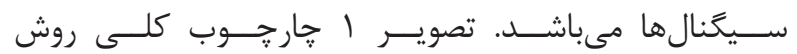

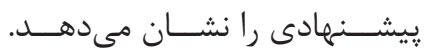

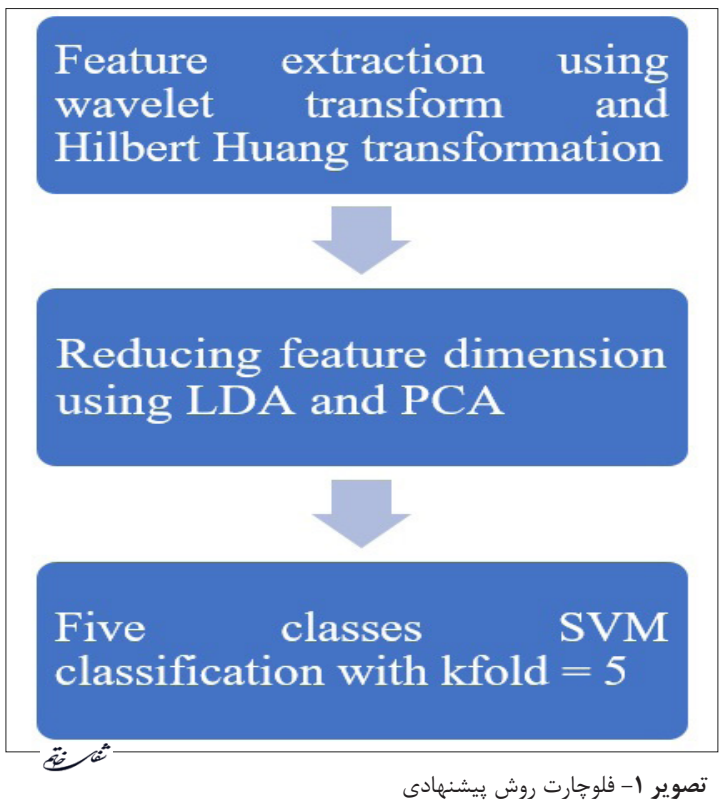

انتخــاب موجـك مناسـب و تعـداد سـطوح تحليلـى در آناليـز

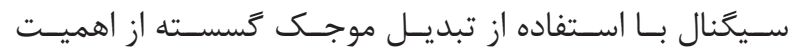

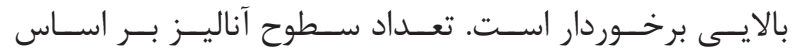

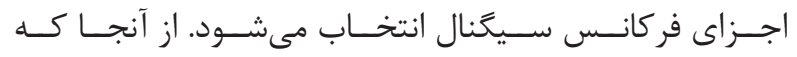

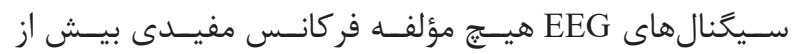

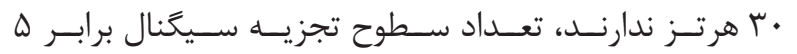

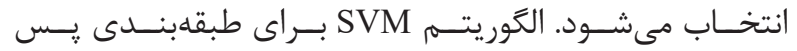

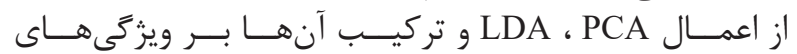

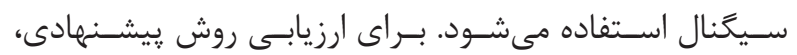

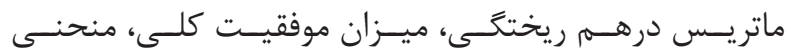

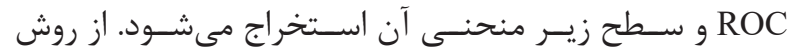

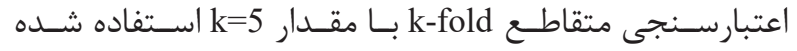

بافتهها.

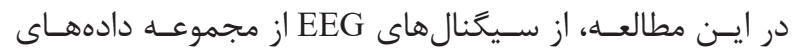

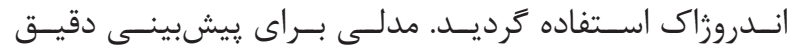

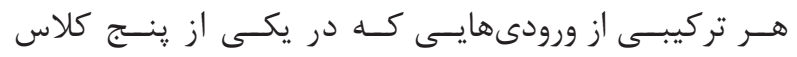

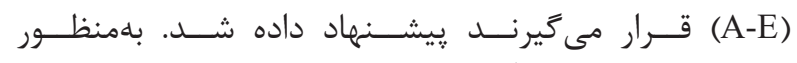

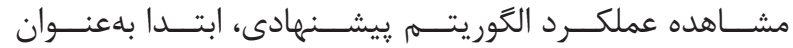

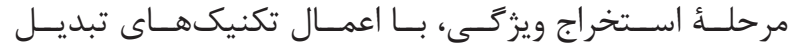

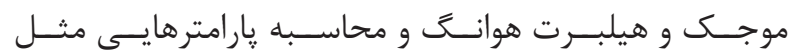

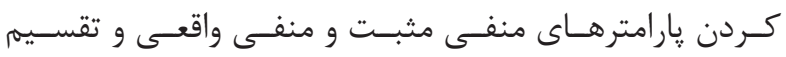

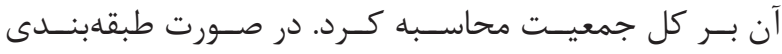

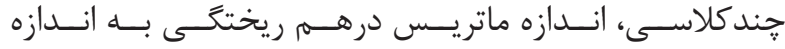

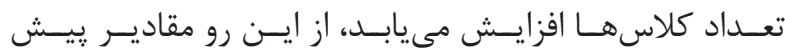

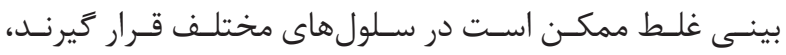

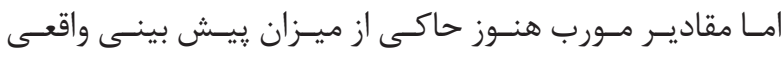

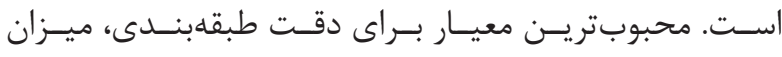

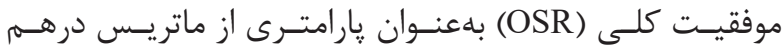

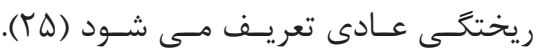

\section{منحنى مشخصةُ عملكرد سيسته}

ايـن منحنــى كـهـ بــا رسـم ميـزان مثبـت واقعسى (حساسـيت)

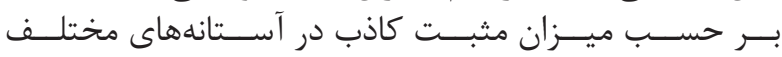

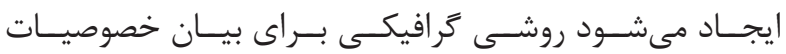

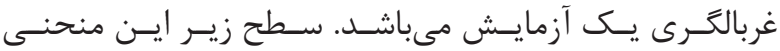

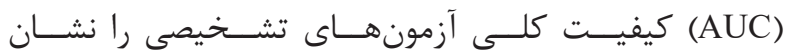

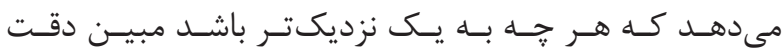

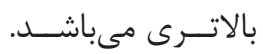

\section{اعتبار سنجى متقاطع}

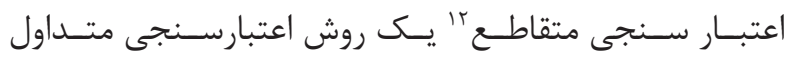

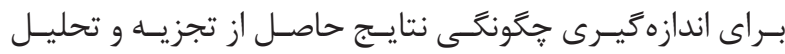

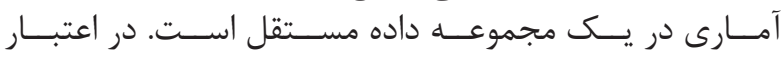

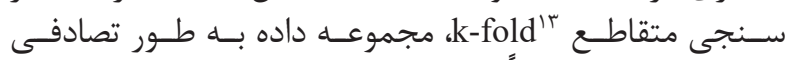

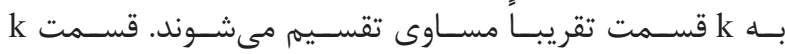

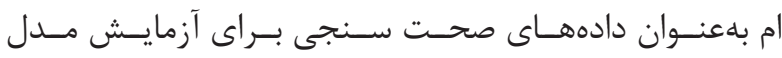

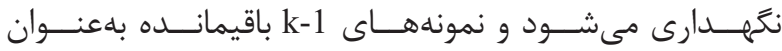

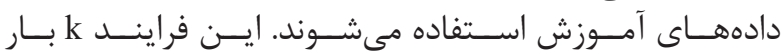

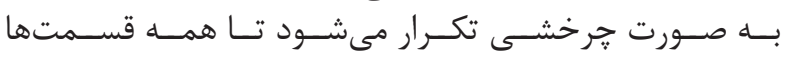

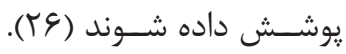

\section{روش يِيشنهادى}

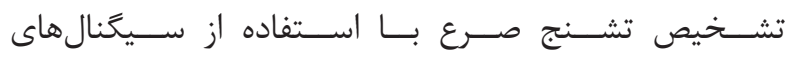

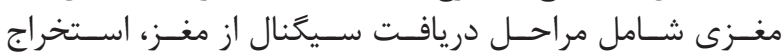

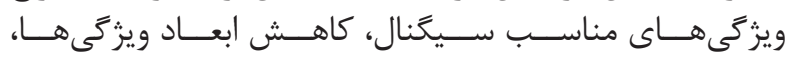

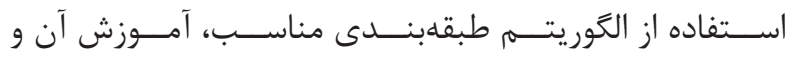

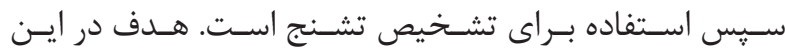

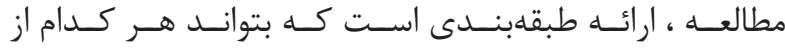

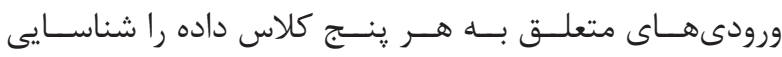

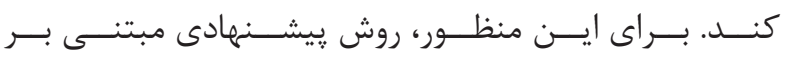

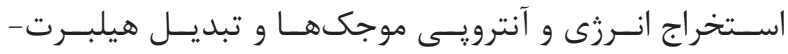

${ }^{12}$ Cross validation

${ }^{13} \mathrm{~K}$-fold cross validation 


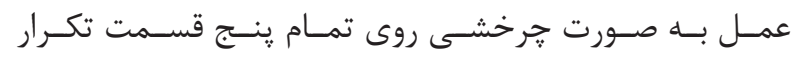

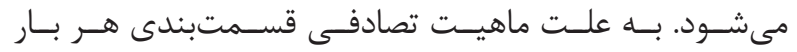

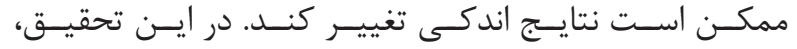

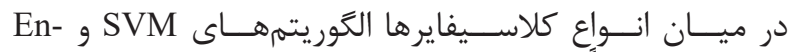
semble

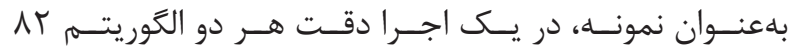

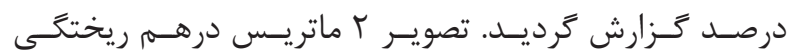

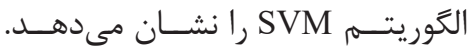

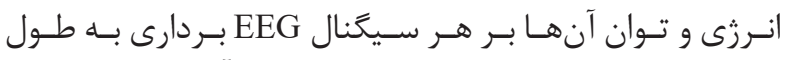

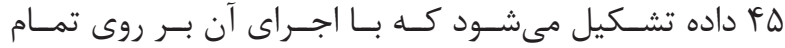

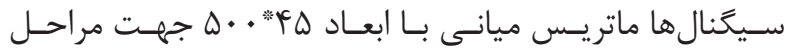

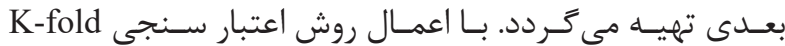

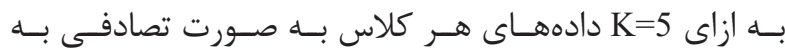

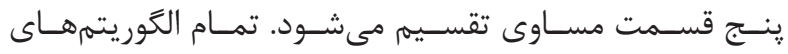

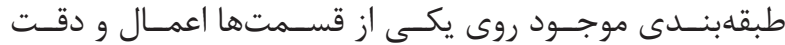

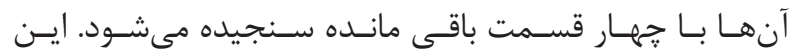

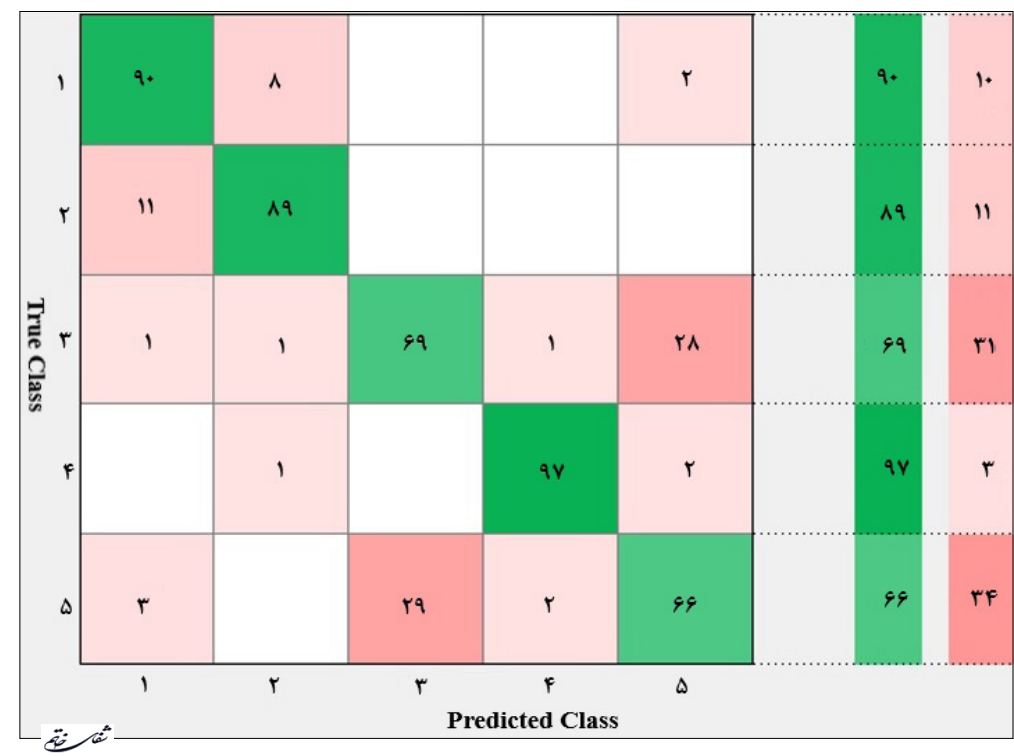

تصوير r- ماتريس درهم ريختىى حاصل اجراى الكوريتم SVM بدون كاهش ابعاد SVM بدون كاهش ابعاد

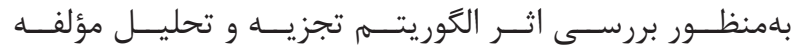

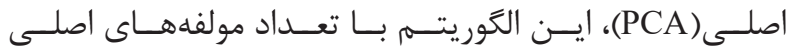

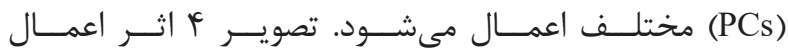

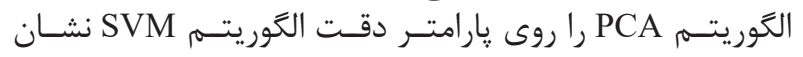

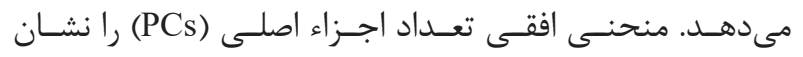

$$
\text { مى دهـــد. }
$$

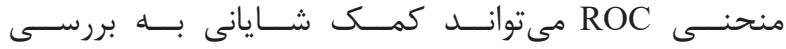

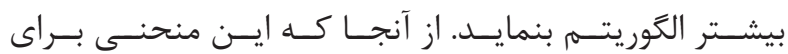

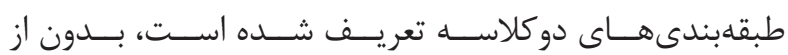

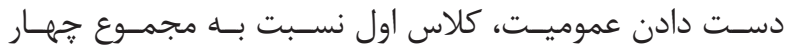

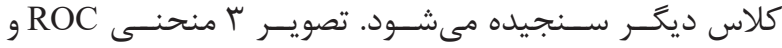

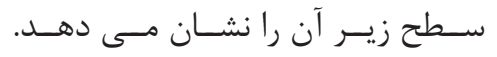

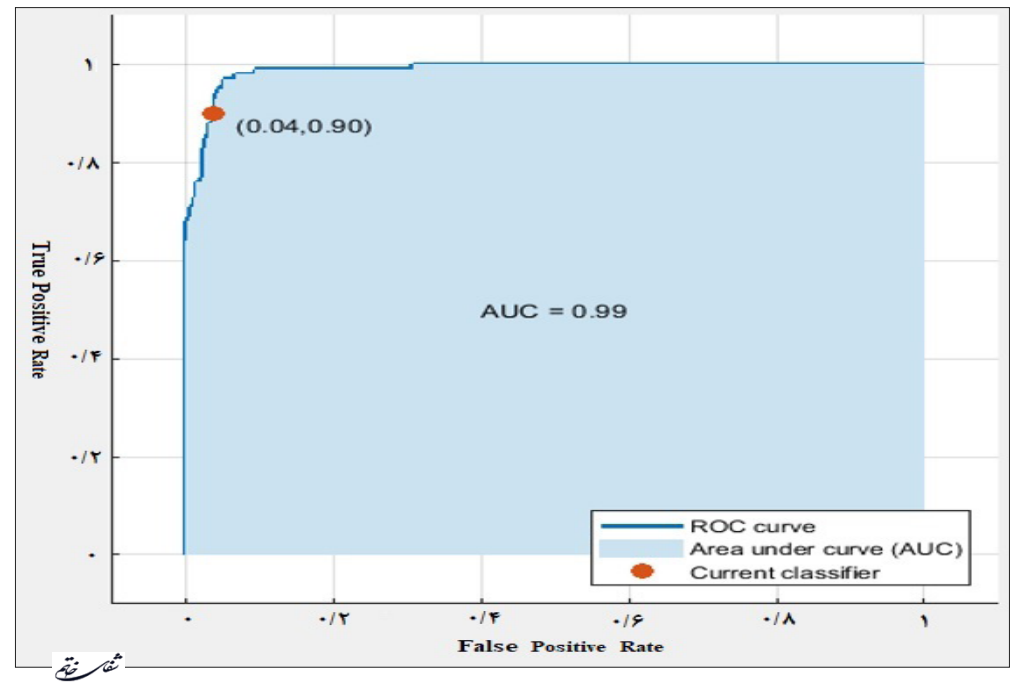

تصوير بـ- منحنى ROC حاصل از اجراى الكوريتم SVM بدون كاهش ابعاد 


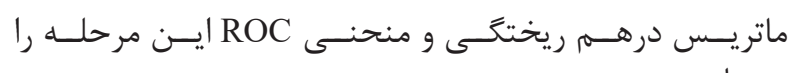

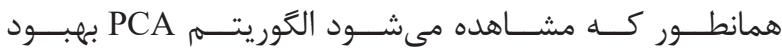

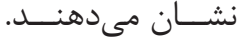

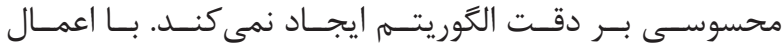
بحث و نتيجه كَيرى

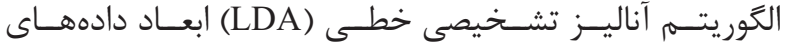

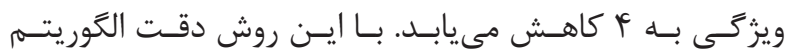

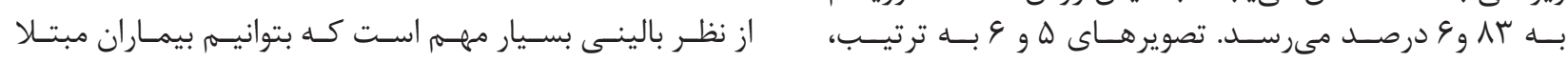

\section{SVM Accuracy}

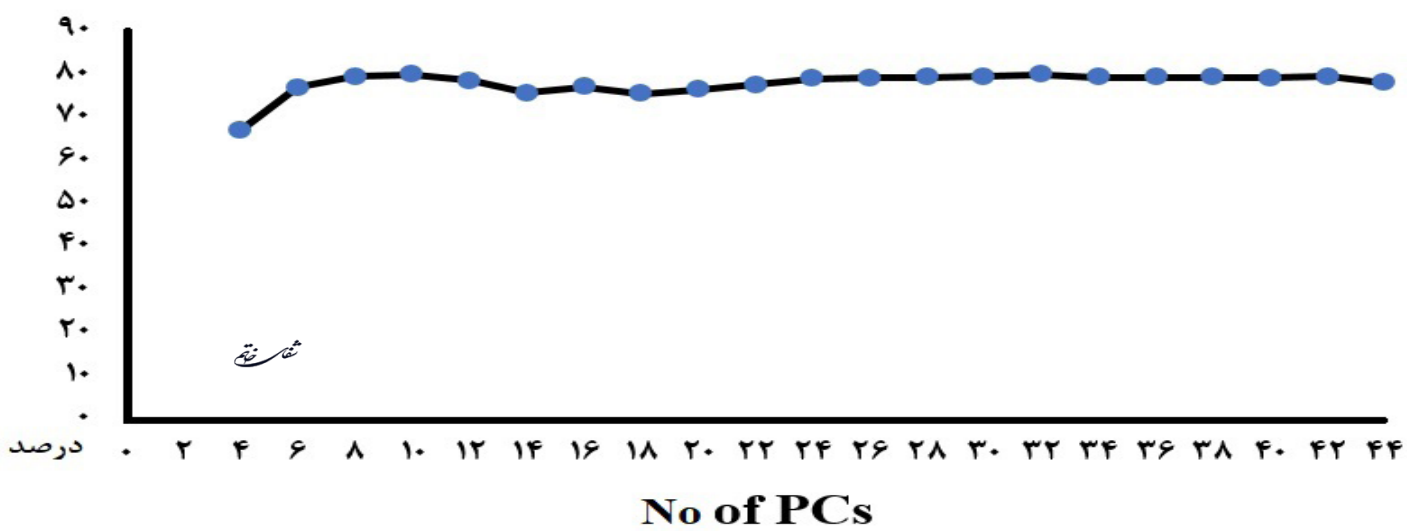

تصوير ץ- اثر اعمال الكَوريتم PCA روى بارامتر دقت الكَوريتم

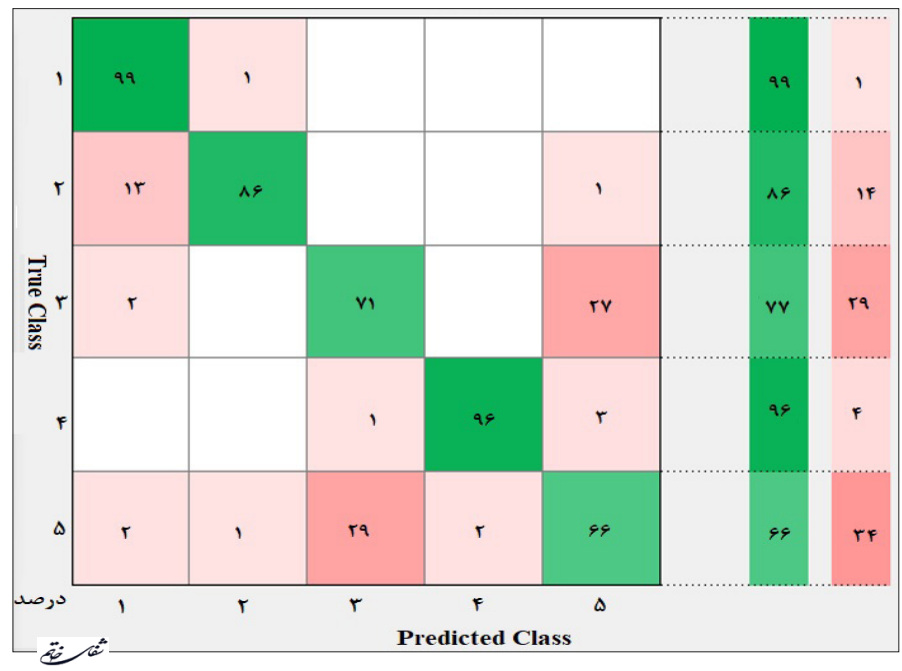

تصوير ه- ماتريس درهم ريختكى با اعمال الكَوريتم LDA

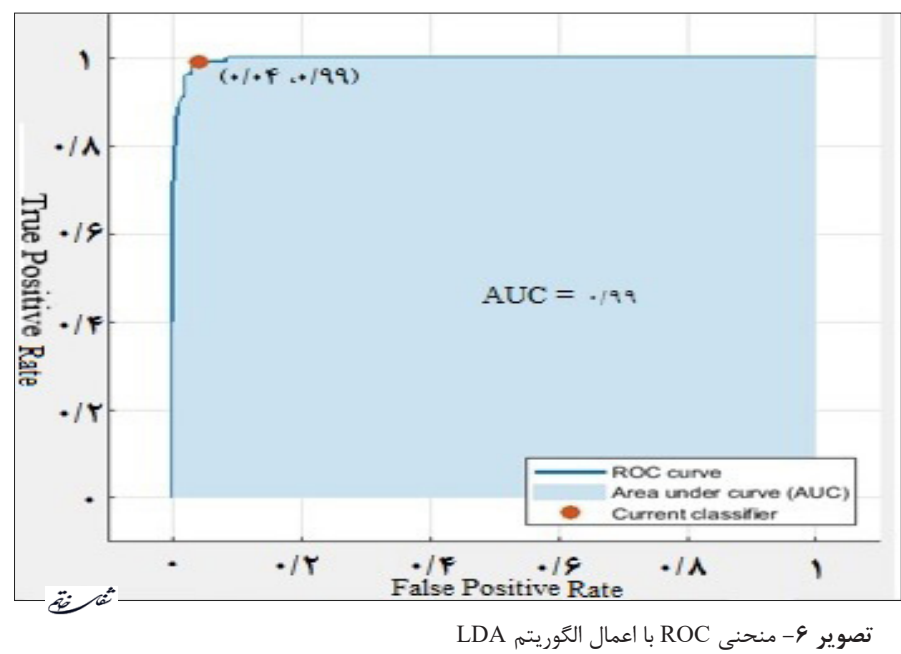




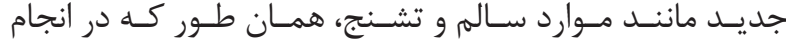

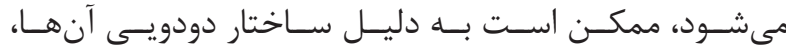

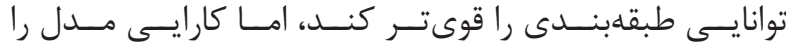

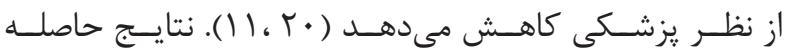

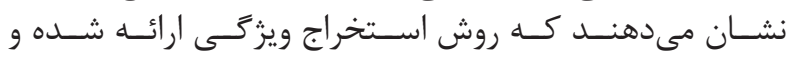

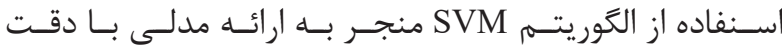

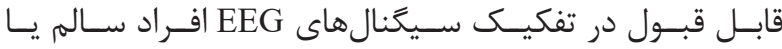

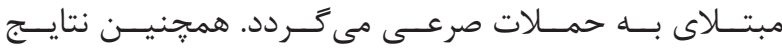

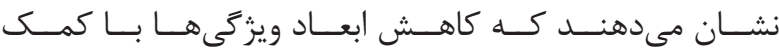

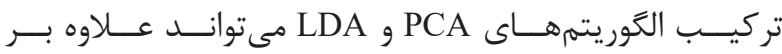

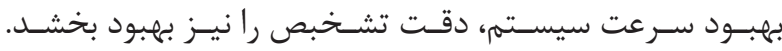

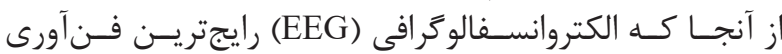

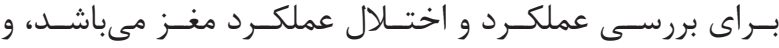

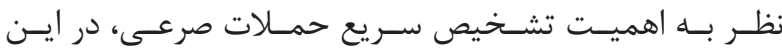

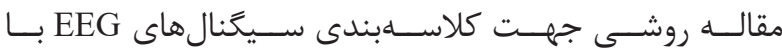

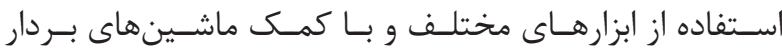

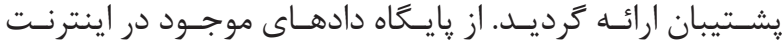

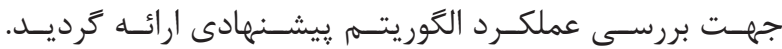

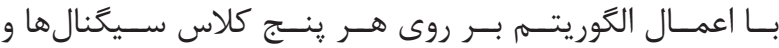

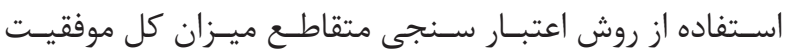

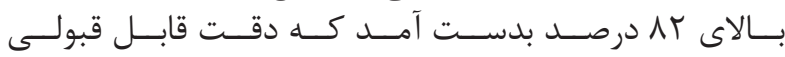

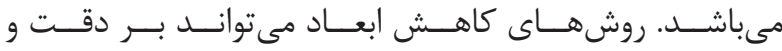

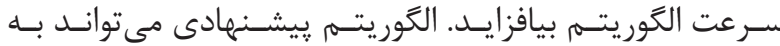

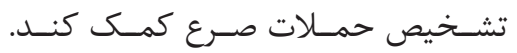

1. Litt B, Echauz J. Prediction of epileptic seizures. The Lancet Neurology. 2002 May 1; 1(1): 22-30.

2. Asadi-Pooya AA, Nikseresht AR, Yaghoobi E, Nei M. Physical injuries in patients with epilepsy and their associated risk factors. Seizure 2012; 21: 165-68.

3. Asadi-Pooya AA, Sperling MR. Clinical features of sudden unexpected death in epilepsy. J Clin Neurophysiol 2009; 26(5): 297-301.

4. Bronzino JD. Principles of electroencephalography. The biomedical engineering handbook. 1995;1.

5. Doppelbauer A, Zeitlhofer J, Zifko U, Baumgartner C, Mayr N, Deecke L. Occurrence of epileptiform activity in the routine EEG of epileptic patients. Acta neurologica scandinavica. 1993 May; 87(5): 345-52.

6. Subasi A. EEG signal classification using wavelet feature extraction and a mixture of expert model. Expert Systems with Applications. 2007 May 1; 32(4): 1084-93.

7. Bashar MK, Reza F, Idris Z, Yoshida H. Epileptic

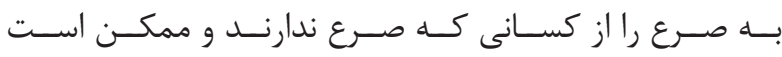

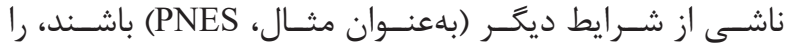

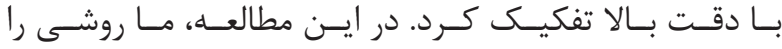

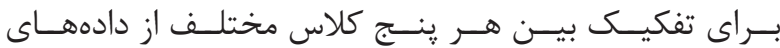

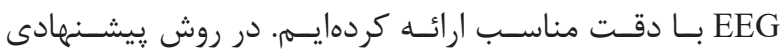

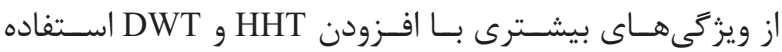

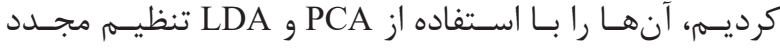

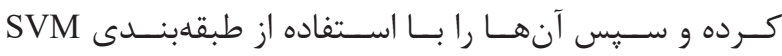
تفكيـك كرديسم.

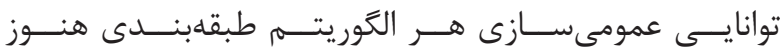

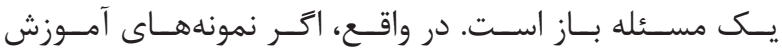

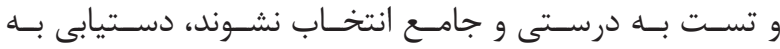

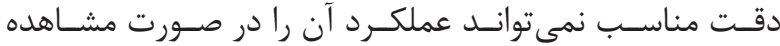

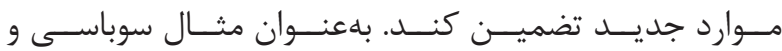

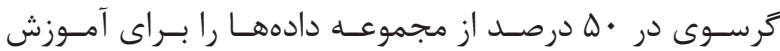

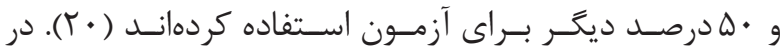

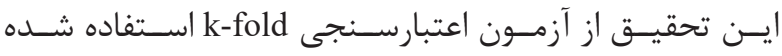

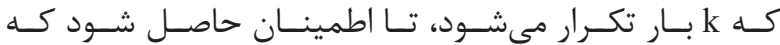

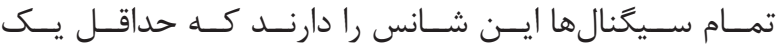

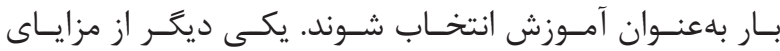

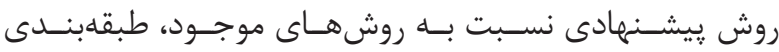

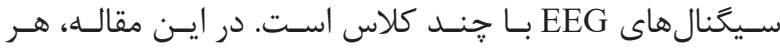

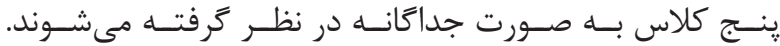

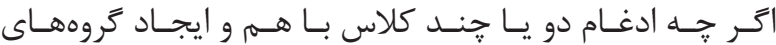

منابع

seizure classification from intracranial EEG signals: A comparative study EEG-based seizure classification. InBiomedical Engineering and Sciences (IECBES), 2016 IEEEEMBSConference on2016Dec4(pp.96-101).IEEE.

8. Gupta A, Singh P, Karlekar M. A Novel Signal Modeling Approach for Classification of Seizure and Seizure-Free EEG Signals. IEEE Transactions on Neural Systems and Rehabilitation Engineering. 2018 May; 26(5): 925-35.

9. Li Y, Wang XD, Luo ML, Li K, Yang XF, Guo Q. Epileptic seizure classification of EEGs using time-frequency analysis based multiscale radial basis functions. IEEE journal of biomedical and health informatics. 2018 Mar; 22(2): 386-97.

10. Correa AG, Laciar E, Orosco L, Gomez ME, Otoya R, Jane R. An energy-based detection algorithm of epileptic seizures in EEG records. InEngineering in Medicine and Biology Society, 2009. EMBC 2009. Annual International Conference of the IEEE2009Sep 3 (pp. 1384-1387). IEEE.

11. Oweis RJ, Abdulhay EW. Seizure classification 
in EEG signals utilizing Hilbert-Huang transform. Biomedical engineering online. 2011 Dec; 10(1): 38.

12. Jiang $\mathrm{Y}, \mathrm{Wu} \mathrm{D}$, Deng $\mathrm{Z}$, Qian $\mathrm{P}$, Wang J, Wang G, Chung FL, Choi KS, Wang S. Seizure Classification from EEG Signals using Transfer Learning, Semi-Supervised Learning and TSK Fuzzy System. IEEE Transactions on Neural Systems and Rehabilitation Engineering. 2017 Dec; 25(12): 2270-84.

13. Fasil OK, Rajesh R. Time-domain exponential energy for epileptic EEG signal classification. Neuroscience Letters. 2019 Feb 16; 694: 1-8

14.JothirajSN,SelvarajTG, RamasamyB,DeivendranNP, Subathra MS. Classification of EEG signals for detection of epileptic seizure activities based on feature extraction from brain maps using image processing algorithms. IET Image Processing. 2018 Aug 14; 12(12): 2153-62.

15. Andrzejak RG, Lehnertz K, Mormann F, Rieke C, David P, Elger CE. Indications of nonlinear deterministic and finite-dimensional structures in time series of brain electrical activity: Dependence on recording region and brain state. Physical Review E. 2001 Nov 20; 64(6): 061907.

$16 . \mathrm{https}: / /$ archive.ics.uci.edu/m $1 /$ datasets/Epileptic + Seizure+Recognition

17. Guo L, Rivero D, Seoane JA, Pazos A. Classification of EEG signals using relative wavelet energy and artificial neural networks. InProceedings of the first ACM/SIGEVO Summit on Genetic and Evolutionary Computation 2009 Jun 12 (pp. 177-184). ACM.

18. Acharya UR, Molinari F, Sree SV, Chattopadhyay $\mathrm{S}, \mathrm{Ng} \mathrm{KH}$, Suri JS. Automated diagnosis of epileptic EEG using entropies. Biomedical Signal Processing and Control. 2012 Jul 1; 7(4): 401-8.

19. Ocak H. Optimal classification of epileptic seizures in EEG using wavelet analysis and genetic algorithm. Signal processing. 2008 Jul 1; 88(7): 1858-67.
20. Subasi A, Gursoy MI. EEG signal classification

using PCA, ICA, LDA and support vector machines. Expert systems with applications. 2010 Dec 1;37(12):8659-66. [17] Mallat, S. (1999). A wavelet tour of signal processing. Elsevier.

21. Huang NE, Shen Z, Long SR, Wu MC, Shih HH, Zheng Q, Yen NC, Tung CC, Liu HH. The empirical mode decomposition and the Hilbert spectrum for nonlinear and non-stationary time series analysis. InProceedings of the Royal Society of London A: mathematical, physical and engineering sciences 1998 Mar 8 (Vol. 454, No. 1971, pp. 903-995). The Royal Society.

22. Lin $\mathrm{YP}$, Wang $\mathrm{CH}$, Wu TL, Jeng $\mathrm{SK}$, Chen JH. Support vector machine for EEG signal classification during listening to emotional music. InMultimedia Signal Processing, 2008 IEEE 10th Workshop on 2008 Oct 8 (pp. 127-130). IEEE.

23. Richhariya B, Tanveer M. EEG signal classification using universum support vector machine. Expert Systems with Applications. 2018 Sep 15; 106: 169-82.

24. Murugavel AM, Ramakrishnan S. Hierarchical multi-class SVM with ELM kernel for epileptic EEG signal classification. Medical \& biological engineering \& computing. 2016 Jan 1; 54(1): 149-61.

25. https://en.wikipedia.org/wiki/Sensitivity_and specificity, accessed 28 Jan 2019.

26. Labatut V, Cherifi H. Evaluation of performance measures for classifiers comparison. arXiv preprint arXiv:1112. 4133. 2011 Dec 18

27. Polat K, Güneş S. Classification of epileptiform EEG using a hybrid system based on decision tree classifier and fast Fourier transform. Applied Mathematics and Computation. 2007 Apr 15; 187(2): 1017-26.

28. Asadi-Pooya AA, Sperling MR Epidemiology of psychogenic non-epileptic seizures. Epilepsy Behav 2015; 46: 60-65. 